\title{
Composing for a latency-rich environment
}

\author{
Michael Rofe | Erik Geelhoed
}

\section{Abstract}

Three new works were commissioned for the pilot performance of Online Orchestra: In Sea-Cold Lyonesse by John Pickard, Spiritus Telecommunitas by Federico Reuben and Re-Tracing by Jim Aitchison. This article brings together post-project interviews with composers and detailed analyses of the final compositions in order to document how composers approached the challenge of writing music for a latency-rich environment. Solutions include the use of distributed textures, polyrhythm and ostinati, blurred transitions, slow rates of harmonic change, layered textures and semi-improvised rhythmic notation. This leads to consideration of the medium of Online Orchestra - a telematic performance environment for distributed young and amateur musicians to make music together - and the conclusion that latency-rich environments hold significant potential for composers.

\section{Keywords}

Online Orchestra; telematic performance; composition; latency; Jim Aitchison; John Pickard; Federico Reuben 


\section{Introduction}

As part of the Online Orchestra research project, three new works were commissioned for a pilot performance that took place in July 2015, involving musicians in four locations around Cornwall, United Kingdom (see Rofe et al. 2017 for an overview of the project). The brief was to compose a work lasting around ten minutes, to be performed telematically by distributed young and amateur musicians. The telematic ensemble would be formed of string orchestra and female vocal group in Truro Cathedral, a brass band in Mullion on the Lizard peninsula and a flute choir at Five Islands' School, Isles of Scilly; a single conductor at Falmouth University would lead the whole ensemble.

Composers of varying backgrounds were commissioned, in order (1) to build a team of composers whose combined experiences broadly covered the anticipated challenges of the brief and (2) to observe similarities and differences between the ways in which composers responded to that brief in their final works. John Pickard led the team of composers. With a background in orchestral composition and composing for amateur ensembles, Pickard himself had limited previous experience of working with or through technology. Federico Reuben, who was also part of the development team for Online Orchestra's latency-control programme, offered a different set of experiences: his background in sound art and live electronics has dominated his compositional outputs, though having studied under Louis Andriessen he has also composed a range of largescale instrumental works. Jim Aitchison was the only composer to have had previous experience of writing music for a telematic environment: his Portraits for a Study: Music 
After Gerhard Richter was composed for string quartet and Disklavier, the performance of which involved three networked Disklaviers located around the United Kingdom.

As described in Rofe and Reuben, 2017, in this special issue, one of the key challenges for telematic performance is the handling of latency - the delay time involved in processing data and sending/receiving it over the Internet. In order to create a telematic environment that enabled remote communities of musicians to perform from their own locations (rather than travel to specialist institutions), it would not be possible to reduce latency below the threshold at which it becomes perceptible and disruptive to performers (20-30ms): broadband speeds in rural contexts do not have the necessary bandwidth. As such, composers would need to write music for a latency-rich environment: an environment in which latency is present.

This article reports the various musical solutions composers developed to overcome this challenge. Issues discussed are derived from two sources: post-project interviews and analysis of final scores.

\section{Post-project interviews}

Exploratory interviews following the pilot performance were conducted with each composer, in which they were asked to reflect back on their experience. All composers were involved throughout the Online Orchestra project, meeting in bi-monthly working groups to discuss challenges and approaches. As part of these meetings, composers wrote short samples of music that were used by participant musicians to test the emerging system (see Prior et al. 2017; Geelhoed et al. 2017). This in turn acted as an opportunity 
for composers to test musical ideas and how these would behave in a latency-rich environment.

Interviews were unstructured, allowing composers to comment on areas they felt to be of significance, including issues emerging from working groups, and their final approaches to composition. Pickard and Reuben were interviewed in person, each interview lasting approximately two hours. Due to availability, Aitchison instead wrote a short essay on his experiences. Oral interviews were recorded and transcribed, and, along with Aitchison's essay, responses were coded. Different solutions to writing music for latency-rich environment were identified and analysed further, particularly those explored by multiple composers. These recurrent solutions form the basis and structure of this report.

\section{Analysis of scores}

In addition to reporting first-hand perspectives of composers, the authors of this article have additionally inserted and analysed extracts from the three final pieces that exemplify composers' approaches to working in a latency-rich environment. Full scores can be found at www.onlineorchestra.com.

\section{Contexts}

As discussed in Rofe et al. 2017, in this special issue, the history of telematic music has often been driven by composers and composer-technologists, resulting in a wide range of 
different approaches to the medium. Perhaps the most common approach, often as a means of bypassing the challenge of latency, has been to use the telematic environment as a medium for free or semi-structured improvisation, often by highly skilled musicians (Handberg and Jonsson 2005; Kim-Boyle 2008; see Mills 2010 for an overview of improvisational approaches). Other approaches have included: a combination of improvisation and score (Deal and Burtner 2011; Fields 2012); one-way transmission (Cooperstock and Spackman 2001 cited in Driessen et al. 2011; Rowe and Rolnick 2004 cited in Braasch 2009); combining music with other art forms (Cooperstock et al. 1999; Oliveros et al. 2003 both cited in Braasch 2009); mixed reality, involving the overlaying of virtual and live materials (Hamilton et al. 2011; Freeman 2005); or generative music and/or sound art (Chafe and O'Modhrain 1996 cited in Chafe 2009; Yoshida et al. 2005 cited in Driessen et al. 2011; Mizuno 2011; see also Barbosa 2003; Whalley 2015).

Where scores are used, these often employ a significant degree of rhythmic freedom in order to absorb latency. For instance, Min Xiao-Fen's Harmony uses graphic notation to enable temporal flexibility, with instructions to performers such as 'improvise a second time based on the melody, crotchet $=80-96$ ' (in Weaver 2011: 24). Likewise, attempts to perform existing works in a telematic environment have largely been confined to those that enable a certain temporal flexibility. For instance, Terry Riley's In $C$ has seen several telematic performances, enabled by the flexibility of temporal synchronicity in the score. As Hamilton et al. note, 'In $C$ is designed in a manner that lets musical performers move linearly through composed musical cells, choosing repetition and cellonset timings while remaining strictly locked to the underlying metronomic pulse' (2011: 151-2). 
Given that the target users of Online Orchestra would be young and amateur musicians, working group discussions led to the decision to make use of standard notation, in order to avoid the introduction of additional complicating factors for performers. This presented a significant challenge to composers with respect to latency: not only would latency need to be 'written in' to scores, but the musical materials themselves would need to flourish within a latency-rich environment. Before specific solutions to this challenge are explored, it is useful by way of additional context to offer a brief overview of the three works: each was inspired by different aspects of the project, and their overall narrative often influenced composers' approaches to latency; solutions often relate not just to the nature of the medium, but also to the creative content of the music.

\section{In Sea-Cold Lyonesse - John Pickard}

For John Pickard, 'not being a particularly technology-minded person, indeed somebody who is in some ways quite afraid of technology, what I wanted to do was to try and humanize the experience of performing online' (Pickard, interview). This led to a search for a strong underlying narrative to which performers could easily relate. Pickard turned to the legend of the submerged city of Lyonesse: though geographically vague in the early literature in which it appears, the legend eventually came to be associated with the lost Cornish town of Lethowsow, which was believed to lie submerged between Land's End and the Isles of Scilly. The story of Lyonesse has inspired many writers and poets, 
and Pickard's work sets poems by Thomas Hardy ('When I Set Out for Lyonesse') and Walter de la Mare ('Sunk Lyonesse'). As Pickard explains,

The Hardy is an extremely mysterious poem because you don't actually know what the poet experienced at Lyonesse, you just know he goes there and comes back transfigured in some way. So the de la Mare poem for me fills in the gap, where there is this underwater vision: it complemented the Hardy poem. My approach was to use the Hardy as a framing device, enclosing the de la Mare.

(Pickard, interview)

This gives rise to a three-part form, the first and third parts setting the Hardy, with a darker setting of de la Mare in part two. As Pickard notes of this central section, This is the first time in the piece, I think, that you get any sense that there might be something more disturbing below the surface, and then, poetically of course, what we do is dive down into the sea; it takes you into another region.

(interview)

This idea of an underwater vision, as expressed in the de la Mare poem, served to inspire what Pickard describes as an 'underwater piece': 'I mean really this piece is my Cathédrale Engloutie [Debussy]. It is the same legend essentially. Another Breton legend: they're all Celtic myths about submerged cities' (interview). The idea of Lyonesse as a submerged city off the coast of Cornwall had particular resonance in the Online Orchestra project, which saw in its pilot performance musicians on the Isles of Scilly perform with musicians in various locations on the mainland. As Pickard explains, Towards the end of the piece, the brass on the mainland and the flutes on the Isles of Scilly call to each other across the sea, their music conveyed via a fibre-optic cable running across the sea-bed and - who knows? - perhaps through the streets of the submerged town itself. Modern technology and ancient myth meet. 


\section{Spiritus Telecommunitas - Federico Reuben}

For Federico Reuben's Spiritus Telecommunitas, 'the concept was the medium itself' (Reuben, interview). Reuben began by researching the link between music and telecommunications, noting that 'I was really surprised that there are actually so many attempts trying to make music through telecommunications' (Reuben, interview). As Reuben goes on to note, examples include Elisha Gray’s ‘Musical Telegraph’ (1876), which produced sound through simple steel reed oscillators transmitted through a telephone line, and services like the 'Théâtrophone' in Paris (1890-1932), which allowed subscribers to listen to live performances of opera and theatre over telephone lines. References to these, and many other examples of telecommunications, can be found throughout Reuben's work.

Structured in four movements, Spiritus Telecommunitas brings together notated music and free improvisation, with the latter taking place on original telecommunications and early sound recording equipment operated by Aleks Kolkowski, with whom Reuben collaborated in devising the piece. References are made throughout the work to a range of different technologies. Taking movement two as an example, a wax cylinder recording of the first Morse code message ('What hath God wrought?') begins the movement, with its rhythmic pattern then forming the basis of brass materials that follow. Recordings of early telephone transmissions are introduced, leading to a vocal section with text that derives from Alexander Graham Bell's first telephone transmission ('Mister Watson: come here, I want to see you!'). The movement ends with an improvised Telharmonium cadenza: the Telharmonium being the first large-scale electronic synthesizer, and 
predecessor of the Hammond organ, whose sound was transmitted live through telephone lines.

Overall, Reuben sought to explore notions of distance and communication through these references. As he explains,

As a composer, I usually start with the ideas and the concepts: they really help solving the practical compositional issues. I was really interested in the whole idea of telematic listening and listening to something from a distance. Part of the idea of Online Orchestra is listening to ensembles that are not playing in the same space. So it wasn't only about trying to think about how they're going to play, but also how the audience is going to listen to something that is being played from far away. The concept was actually related to the problems of writing a piece for Online Orchestra.

(Reuben, interview)

In enabling remote participants to join together, Reuben is interested in the community-forming potential of telematic music: 'Spiritus Telecommunitas celebrates the spirit of community as enabled through the relationship between music and telecommunications' (interview).

\section{Re-Tracing - Jim Aitchison}

Jim Aitchison, a composer whose music is often written in response to the visual arts, looked to Mark Rothko's work, with its blocks of colour and soft edges, as inspiration for his piece. In 2008, Aitchison had been commissioned to respond in music to Rothko's work, as part of a major exhibition at the Tate Modern. In researching that work, Aitchison discovered from conversations with the exhibition curator, Achim Borchardt- 
Hume, that Rothko visited Cornwall in 1959 and wrote to Cornish-based artist Patrick Heron shortly following this visit describing his intention to return the money for the Seagram commission: one of the largest commissions, and one of the most celebrated withdrawals, in art history; it seems that Heron was one of the first people to have been informed.

As Aitchison describes, his previous work for distributed performers, Portraits for a Study: Music After Gerhard Richter, had already explored 'general themes of absence, distance, travel, and change resulting from passage through some kind of threshold', and this resulted in 'a line, or lines, that lacked definition and tended towards being blurred' (interview). These principles found expression in Re-Tracing, as applied to Rothko's journey to Cornwall. As Aitchison explains,

I traced Rothko's journey from New York, across the Atlantic, to Naples, then to Florence and up through Europe and eventually to Cornwall. The map contour was placed upon manuscript paper, which gave a simple melodic shape, and the place names a text to be sung.

(interview)

Figure 1 charts this journey and shows Aitchison's own tracing of this line onto manuscript, segmented into three parts. These lines form the basis of linear melodic gestures throughout the work, as discussed below. 
Figure 1: Rothko's journey and its musical tracing. $]^{[}$

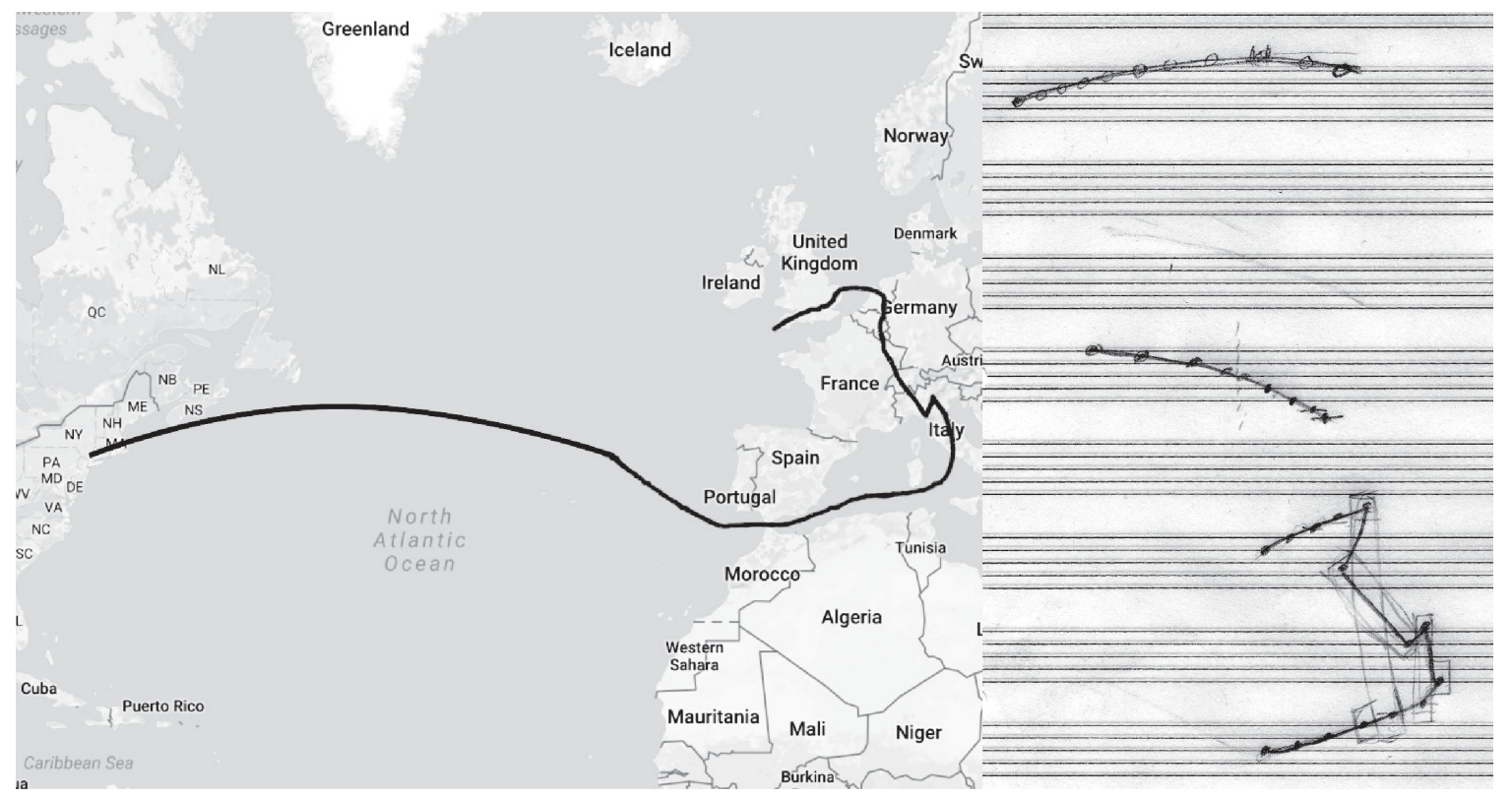

For Aitchison,

Rothko returned to the US from Cornwall having made a momentous decision that changed his life, and art history. Accordingly, I decided to retrace his journey in retrograde, but this time on the surface of the Moon, over the empty seas of the lunar landscape.

(interview)

This enabled a two-part form within the piece overall, with linear gestures in the second part presented in retrograde; the text of part two charts place names from the lunar surface. As Aitchison goes on to explain, 'My decisions regarding how to affect the melodic material derived from the artist's route came directly from the conditions emanating from the Online Orchestra system, and how these resonated with my understanding of aspects of Rothko's artistic procedures' (interview). In particular, Rothko's 1934 statement that he sought the '[...] simple expression of the complex thought' (Rothko 1934: X7), served as an important source of inspiration, resulting in a 
slowly evolving soundmass, derived from the overlaying of multiple versions of the melodic line (discussed below).

\section{Latency control}

As described in Rofe and Reuben, 2017, in this special issue, Online Orchestra's overall approach was to control latency, rather than let it roam freely as a consequence of network conditions. As such, developers designed a programme that (1) removes shortterm variation in latency in order to establish a stable value, (2) unifies the latency within a multi-nodal architecture, such that latencies between all nodes are the same, and (3) locks that latency to a desired musical tempo, such that the duration of the latency is equivalent to the duration of a musical beat (users can specify this duration in order to enable different tempi).

So whilst the Online Orchestra environment is latency rich, that latency is predictable. Specifically, composers knew that a note performed in one node would be heard exactly one beat later in other nodes, and this provided a rule system within which to compose music: in effect, composers needed to write the latency into the scores, such that when performed in a latency-rich environment, the music would sound as intended. This situation is rendered more complex, though, as additional nodes are added to the system. For instance, in a three-node orchestra where the score indicates that all musicians should play a note on the downbeat: from node one's perspective, they play on the downbeat, but hear nodes two and three a beat later; from node two's perspective, they play on the downbeat, and it is nodes one and three who sound a beat later; likewise 
in node three, sound from nodes one and two are a beat later. In other words, given a single score there are as many different sounding versions of that score as there are nodes in the orchestra.

These conditions present a significant challenge to the composer, who must take account not only of the latency displacement, but also write music that makes sense in multiple sonic versions. During the planning stages, prior to the development of the final latency-control programme, composers discussed this challenge and considered alternatives to rhythmically precise music. As Reuben notes, 'One of the first considerations was that it would be very difficult to enable synchronicity between musicians. I had ideas of writing pieces that wouldn't require that type of synchronicity' (interview). However,

As we progressed with the technical solutions, it became apparent that it was actually possible to solve that by stabilizing the latency, and then it was possible to play with the idea of musical synchronicity, which was obviously very exciting in that we were not able to do that before.

(Reuben, interview)

Pickard likewise agrees that the controlling of latency was a crucial step in enabling work to progress:

The moment that we had the program, that was the real break-through; the fact that we knew what we were going to get. Up to that point, we knew that the latency might roam freely and it might vary. That didn't worry me unduly, but I must admit I was a lot happier when we stabilized it.

(interview)

Crucially, the stabilizing of latency unlocked a new range of compositional options. Where Reuben began by considering possibilities for non-synchronous music, 
the programme enabled greater confidence to explore a wider range of interlocking musical textures. As Aitchison notes, the programme meant that 'it was possible to calculate the latency effect at each node, and plan the compositional events accordingly with real accuracy, down to a local level of detail' (interview). As such, as Pickard points out, 'there was no difference in writing this piece than in writing any other piece. The only issue was about the simultaneity of events' (interview).

What remained, though, was the challenge of writing music that would be acceptable in multiple manifestations, given the varying latency relationships between nodes, as outlined above. A notional solution could have been to prioritize the version in one node at the expense of others: to displace musical content in three nodes, such that the music would sound 'correct' in the fourth. However, this approach would go against a primary aim of the project, which was to enable a meaningful and potentially educative experience for all musicians in the ensemble, not just those in one of its locations. As such, it would be necessary instead to write music with the necessary flexibility to enable all sonic versions to sound acceptable. As Reuben notes,

That I think was the main challenge. When you're writing music, usually the score reflects how it's supposed to sound in one place. But in this situation you had to write the music knowing that it was not going to sound that way in most of the locations.

(interview)

In order to assist with this challenge, Online Orchestra developers created a second programme that takes midi data, as exported from notation software such as Sibelius, and displaces the content, such that the composer can hear how the music will sound in each node given the latency. As such, composers were able to produce a single score, but knew precisely how this score would be realized sonically in its different 
manifestations. This presents an interesting ontological question concerning what constitutes 'the piece', as Pickard explains:

I think they're all right versions, although I would say that the version that we have in the score is probably 'righter'. Isn't it great? A wonderful irony, isn't it? The only people to hear that version were the conductor and me.

(interview)

As outlined above, the three final compositions were highly diverse in their aesthetic, content and structure. However, in order to find compositional solutions to the challenge of latency, a number of techniques common to all pieces can be seen.

\section{Distributed textures}

A simple solution to composing for latency-rich environments is to distribute a texture across the ensemble in such a way that rhythmic interaction takes place within isolated nodes, rather than between nodes. Figure 2 shows an example of this type of distributed texture, taken from Pickard's In Sea-Cold Lyonesse. As can be seen, focus moves from the string and vocal materials of Node 1 to flutes in Node 2, with the C-D of the strings imitated in the flutes to smooth the transition. From b58, flutes have highly active materials, but this rhythmic activity does not spread to other parts of the ensemble: strings now take on an accompanimental role, with long notes that do not require precise rhythmic alignment to any particular note in the flute material. Brass in Node 3 takes over at b61 - again, briefly overlapping with the strings to smooth the transition - but are again confined to long notes in order to foreground the flutes. 
Figure 2: In Sea-Cold Lyonesse - Distributed texture; Bars 56ff.

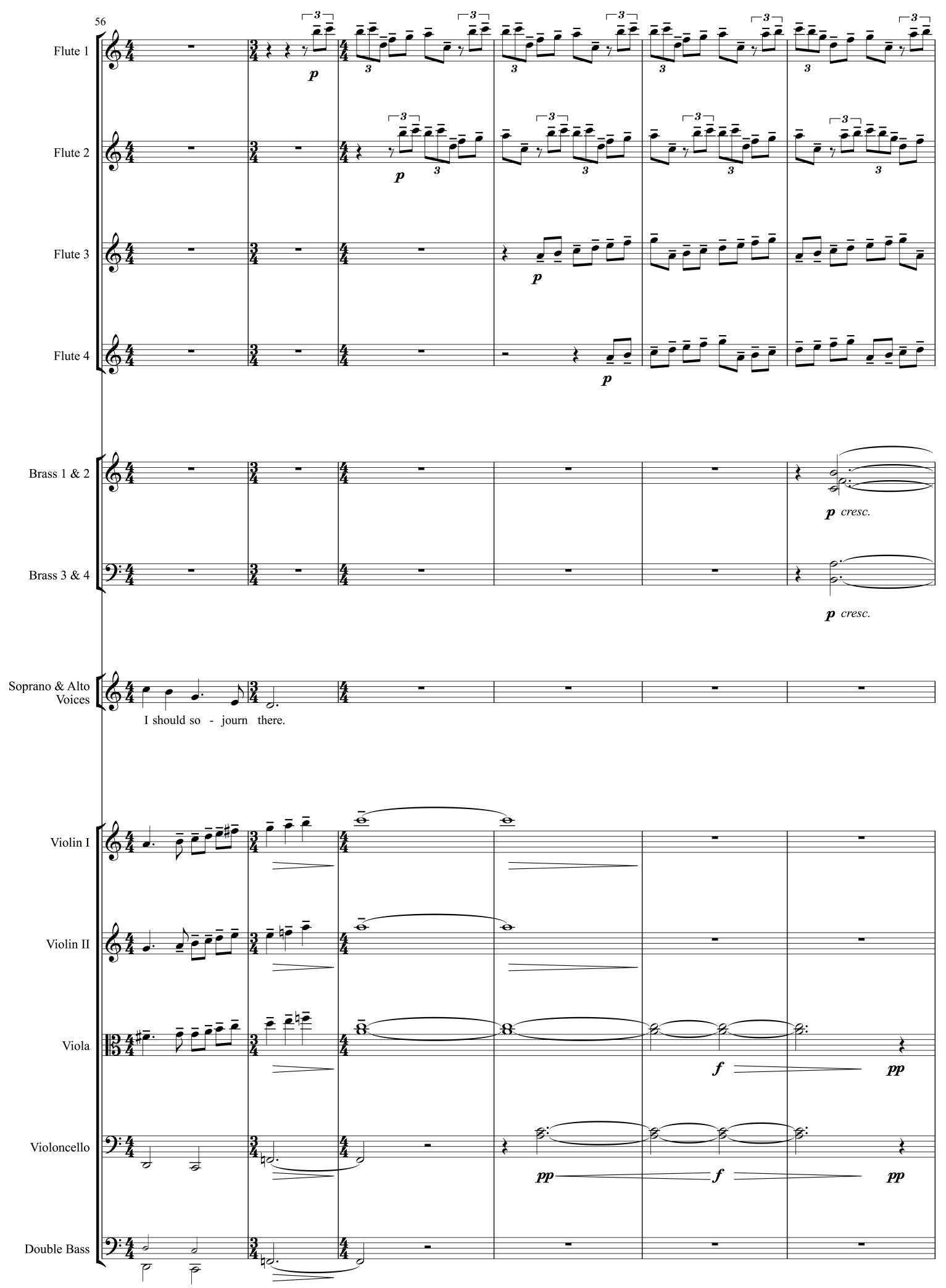


So whilst this is rhythmically active music, that activity is confined to one node at a time. Latency therefore does not disrupt the overall coherence.

Moreover, because the flutes are playing short, and overlapping, repeated phrases, the resultant soundmass can support multiple entry points of the b61 brass chord. From the flute node's perspective, because of the controlled latency in the system the brass actually sound on beat 3 of b61. From the brass node's perspective, the flutes sound one beat later than written, creating a displacement in the other direction. And from the string/vocal node, the entry point of the brass with respect to the flutes is preserved, but all displaced one beat later, with respect to the score. However, in each of these sonic versions, the music makes sense: a swirling flute pattern is supported by a string chord and then a brass chord. As Pickard makes clear, therefore, latency is handled not just by distributing materials, but also by the very nature of the materials themselves: a kind of music is needed 'where there is a certain amount of "give"; a certain amount of tolerance' (interview). This tolerance comes in the flute material: it can support both chords, and it can support the transition between chords at multiple moments.

Figure 3 shows a more complex example of a distributed texture from Pickard's work. On this occasion, not only is rhythmic activity localized within specific nodes at any one time, but similar materials are being passed back and forth between nodes; strings provide a harmonic anchor over which this activity takes place. This passing around of materials serves to obfuscate the presence of latency, but its main function is to connect the distributed musicians: performers located far apart share a connection through the distribution of content. This is discussed in more detail below. 
Figure 3: In Sea-Cold Lyonesse - Sharing of materials; b160ff.

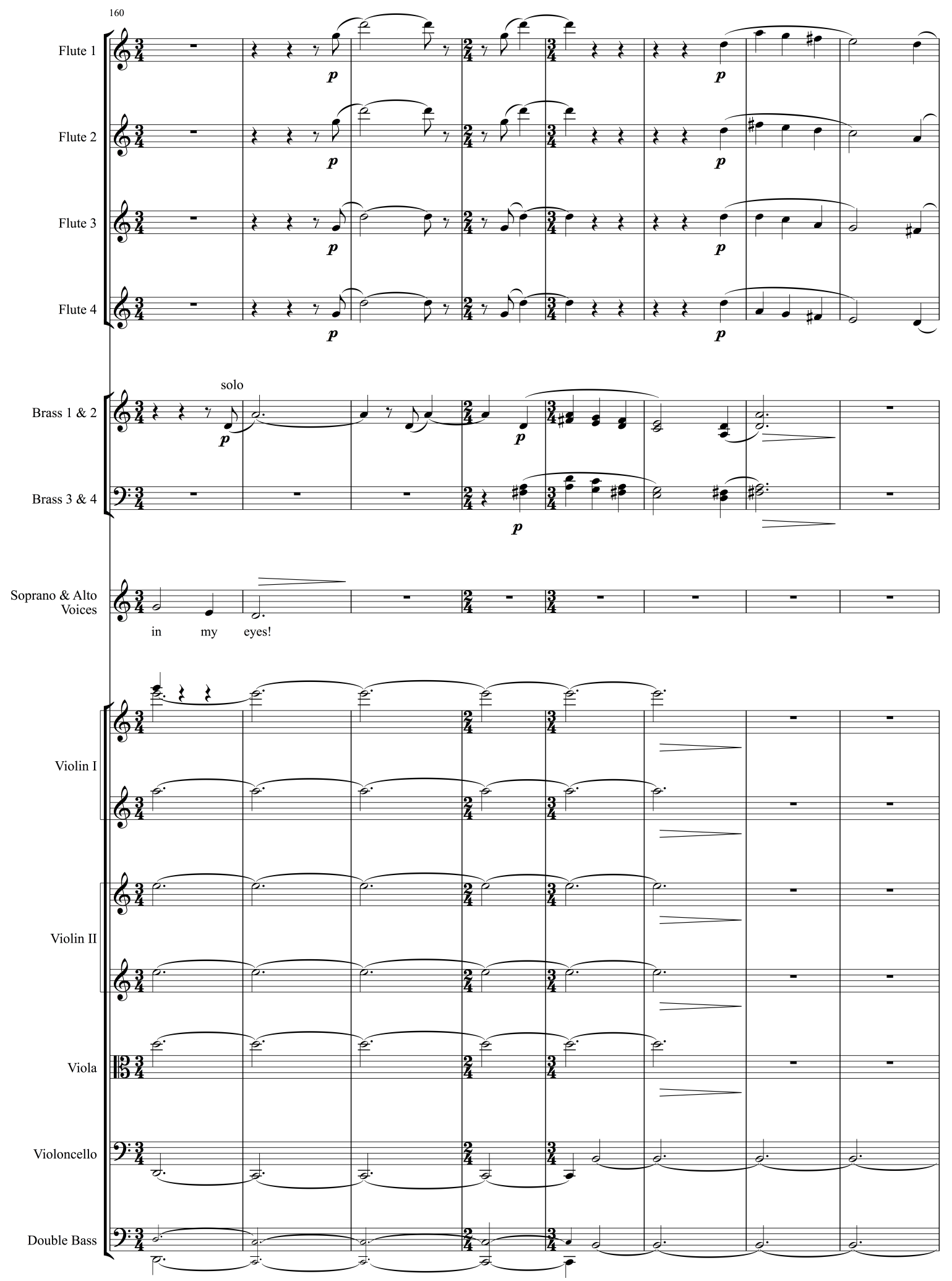


For now, the care taken over the temporal entry points in this imitative passage should be noted. As Pickard describes:

I definitely wanted that sense of them calling to each other. That's quite an important moment $[\ldots]$ What I didn't want was the cornet overlapping with the flutes. And so I had to build in more time, so that they would not be falling over each other. I was OK with a longer gap, but what I didn't want was a sort of musical traffic jam.

(interview)

As such, the slightly elongated cornet of b161 prior to the flute entry ensures that, from the flute node's perspective, they still enter after the cornet, even taking account of the fact that the cornet arrives to them a beat later than written. Again, to use Pickard's term, 'tolerance' is built into the score to absorb the latency.

\section{Polyrhythm and ostinati}

Where examples so far demonstrate rhythmic activity confined to a single node, composers also explored ways of creating precise rhythmic coordination between nodes. As described above, Online Orchestra's latency-control programme locks latency to the desired tempo, such that the duration of a single-trip latency is equivalent to the duration of a musical beat. That locking is highly precise, meaning that complex rhythmic patterns can be realized precisely in time over the network, albeit displaced by a beat. Figure 4 shows an extract from the first movement of Reuben's Spiritus Telecommunitas, demonstrating the extent of rhythmic complexity possible given the latency-control programme. Because the latency is tightly locked to the crotchet beat, semiquavers in turn also lock precisely together, enabling intricate rhythmic relationships between nodes. 
Figure 4: Spiritus Telecommunitas (I) - Inter-nodal rhythms; b112ff.

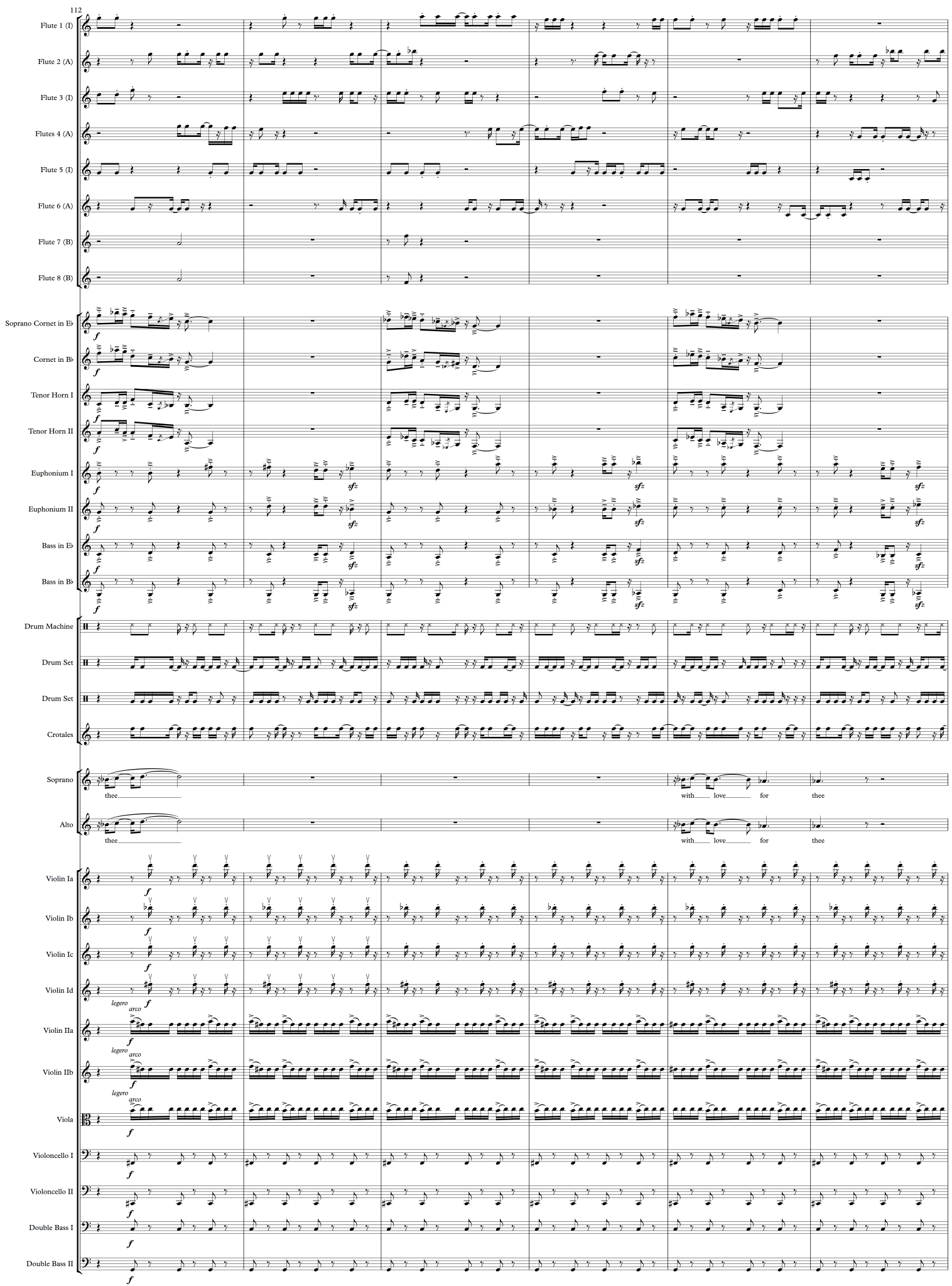


So tight is this locking, in fact, that the piece contains a drum machine part, broadcast through the network from the conductor's node; performers were able to lock their semiquaver rhythms precisely to this pattern.

However, whilst it is possible to build this degree of rhythmic complexity and interactivity, the challenge of enabling multiple sonic versions remains, as latency creates different displacements in each location. In the extract in Figure 4, this is solved by means of a series of layered rhythms and ostinati, whose pattern onset points with respect to each other are of less significance; this is similar to the extract in Figure 2, described above. As Reuben explains, 'the main thing was to find some sort of ostinato type of sound that would lock into the latency tempo, and that would generate layers of rhythm' (interview). In this case, the rhythmic content of the flute materials derives from Morse code, with slowly evolving pitch content that moves these rhythms through a series of harmonies. The string materials derive from spectral analyses of the drum machine sounds; they are, in effect, a string arrangement of a drum kit pattern, which is then overlaid on the drum machine itself. Over these rhythmic layers sit varied repetitions of a brass gesture, forming a foreground to the texture overall.

A consequence of layering complex rhythms in this way is the obfuscation of metre. What emerges is a polyrhythmic texture formed from the interaction of multiple concurrent rhythms and ostinati that flows without reliance on any particular downbeat. This in turn enables different sonic manifestations in each node: for instance, whether the brass gesture sounds on beat one, as it does from the perspective of the brass, or falls on beat two, as it does from the perspectives of flutes and strings, is immaterial. The effect is the same in all locations: a polyrhythmic wash of sound supports a repeating and 
textually dominant brass figure; each node experiences this effect, even though details of its manifestation differ. In any case, from all perspectives, the music appears to be 'in time', despite the latency displacement; all versions sound potentially 'correct'.

For Reuben, this technique was inspired by several significant precedents. Firstly, as Reuben explains, 'being Costa Rican myself I know my Latin American and African rhythms. You have certain kinds of rhythmic patterns that repeat. But sometimes if you shift them a little bit it still works, as long as it's in time' (interview). And, having studied with Louis Andriessen, Reuben also drew inspiration from Minimalist techniques of rhythmic layering and phasing, in order to create complex, contrapuntal textures: 'There is a counterpoint. In a way, what drives the piece is the counterpoint rather than the vertical harmonic language' (interview).

\section{Blurred transitions and slow rates of harmonic change}

This prioritization of linear organization, as opposed to vertical harmonic progression, is a solution to which all composers turned to some extent. The consequences of working in a latency-rich environment most obviously relate to temporal synchronicity and hence rhythm. But, as seen in Figure 4, the latency-control programme actually enables highly complex rhythmic interaction between nodes. In fact, it is the establishment of functional harmonic progression that presents a greater challenge. For instance, Figure 5 shows a simple example of a dominant-tonic cadence, voiced over two parts. But when realized in a latency-rich environment, each node hears a phased version of this cadence, and, more specifically, each node hears a differently phased version. 
Figure 5: Dominant-tonic cadence in a latency-rich environment.

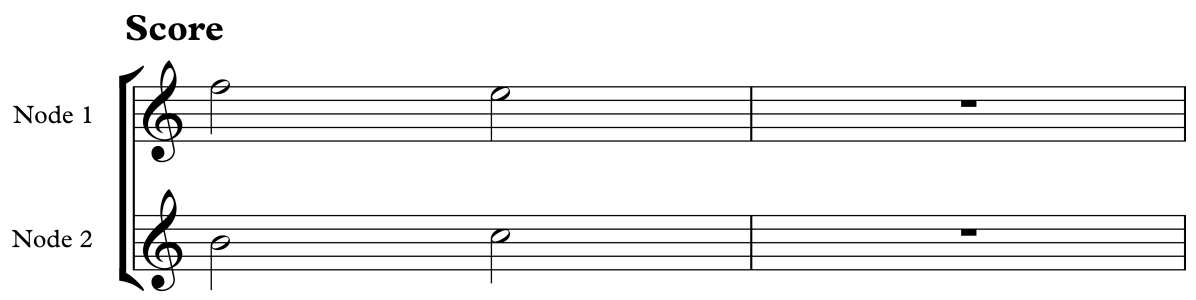

From Node 1's Perspective

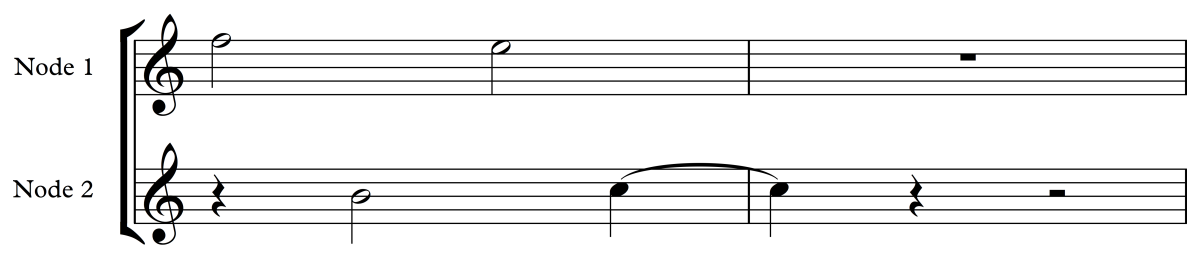

From Node 2's Perspective

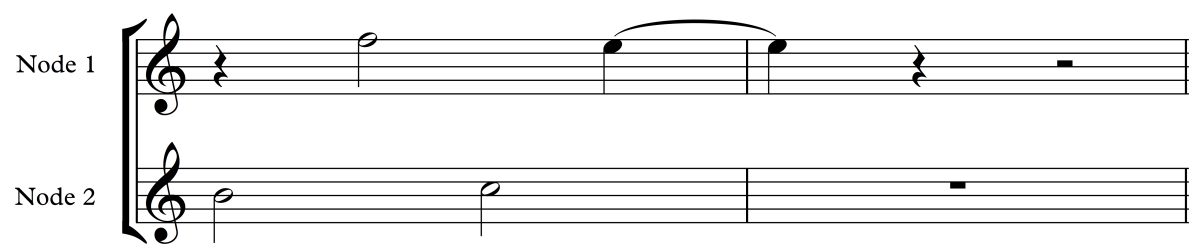

As such, homophonic progressions are realized sonically as a set of suspensions, blurring the boundary between chords.

In the case of Figure 4, the overall harmony is actually quite static, with the only real harmonic movement being effected by the slowly evolving pitch content of the flute and lower brass lines; these details though are rooted over the unchanging strings, creating slowly evolving harmonic colours over an otherwise fixed landscape. As Reuben explains, 'The harmonic language that I was developing works really with suspensions, because if they are a bit late in one place or the other they still work together; things go slightly out of phase and then come back together' (interview). Overall, therefore, 'the harmonic language is quite static and serves more as a timbral effect' (Reuben, interview). 
Figure 6: In Sea-Cold Lyonesse - Blurred harmonic progression; b133ff.

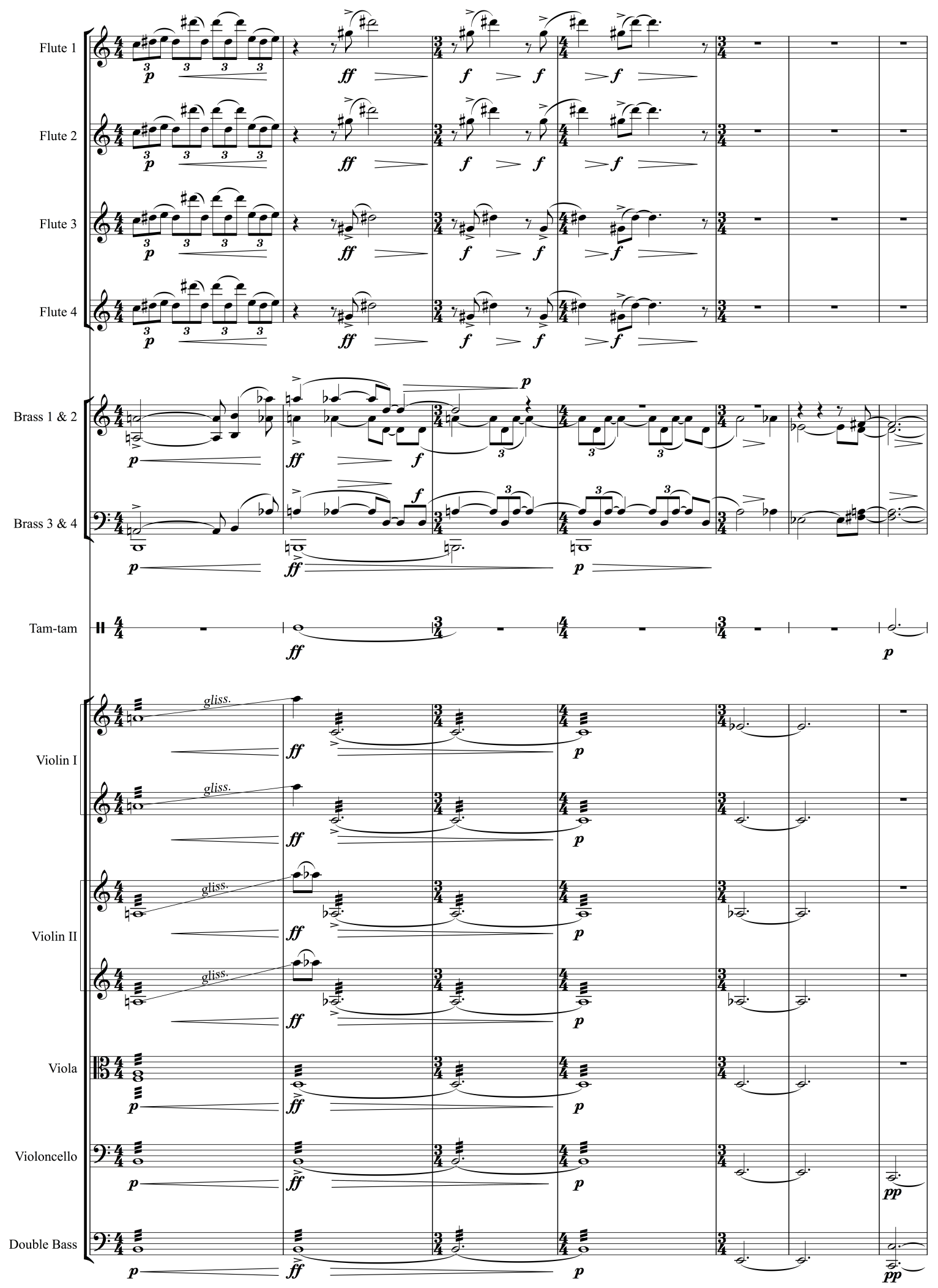


Figure 7: Re-Tracing - Canonic layering; b3ff.

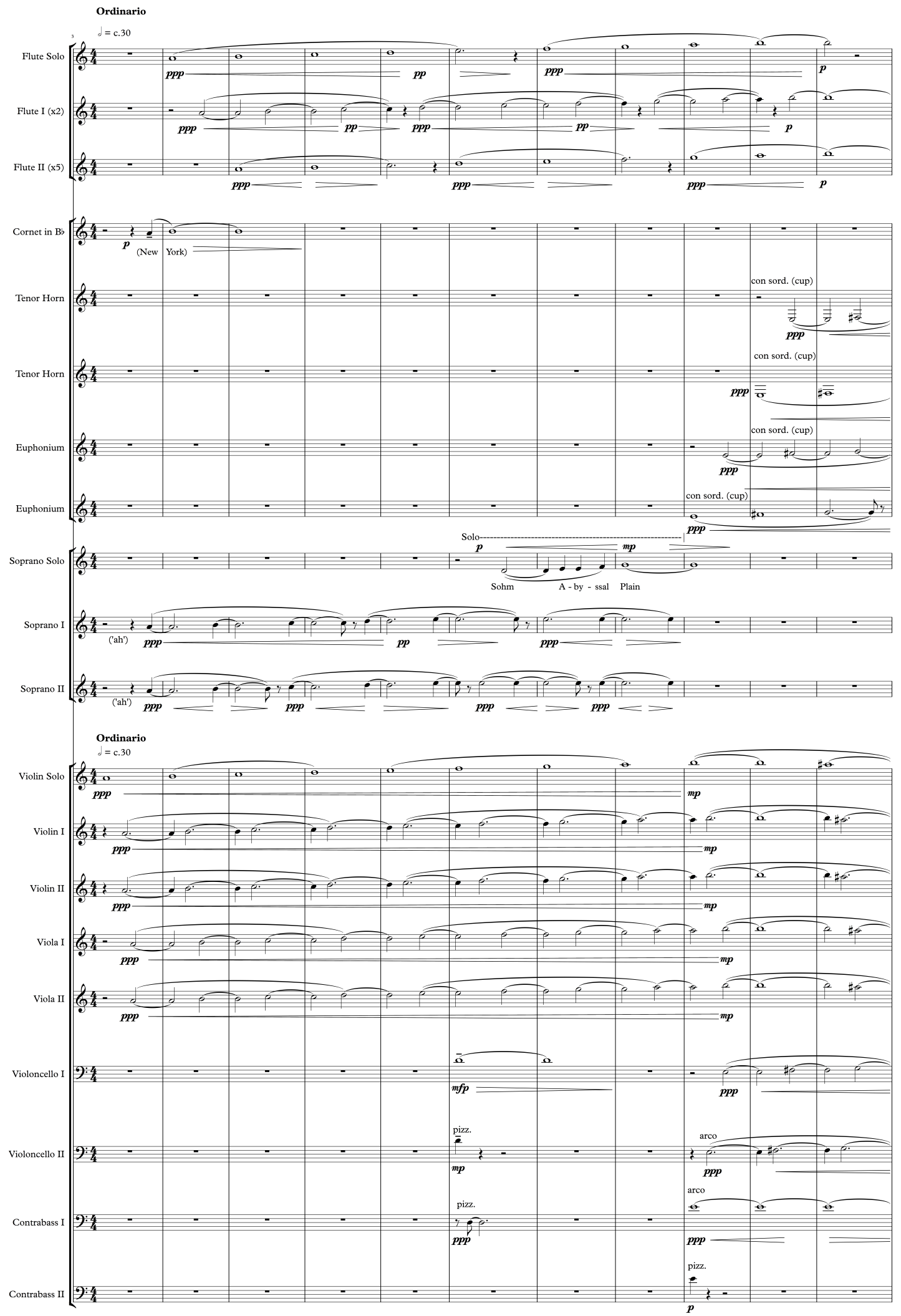


A different solution to the challenge of enabling harmonic progression can be seen in Pickard's work, who interprets the effect shown in Figure 5 in the context of his intention to compose an 'underwater piece', described above. For Pickard, 'If you swim underwater, you basically get a kind of latency. If you're swimming and somebody next to you drops a stone, you hear it after the event because the sound has to carry through the water' (interview). Part of the 'tolerance' that Pickard describes, as detailed above, therefore manifests itself in harmonic progressions that evolve slowly and indeed involve precisely the type of blur between chord changes demonstrated in Figure 5. Figure 6 shows the end of the main climax phase from In Sea-Cold Lyonesse. Here, the D major resolution, as presented in the brass, slowly emerges out of the more chromatic landscape that precedes it, though even here the low $\mathrm{C}$ pedal in the strings maintains a more complex modal sound.

As Pickard explains, There is a slow rate of harmonic change. Also, the way that one chord shifts to another is blurred, so you have these moments where one chord is overlaid on another. There is no doubt that having pedal points definitely anchors the music.

(interview)

For Pickard, a slow tempo is crucial when writing music for a latency-rich environment:

Although I could make the varying latency relationships work in slow music by blurring the outlines, that's going to be far more difficult to do in fast music where the outlines have to be clear; that's what makes it fast. It might work for a certain kind of pulse driven music - a certain kind of Minimalist music - because you can have relatively little harmonic change. But I don't actually consider that to be fast music, I think it is slow music that has a fast beat. When I'm talking about fast music, I'm talking about music 
that has a high rate of harmonic change, has a high rate of contrast, often dynamically, rhythmically and so on.

(interview)

In keeping with Rothko's blocks of colour, Aitchison's approach to harmonic change is likewise slow-paced, but here it combines all elements of the music into a single evolving process. As shown in Figure 7, a musical line - as derived from Rothko's journey in Figure 1 above - forms the basis of all musical materials. As Aitchison explains, this line is explored through a canonic texture:

$[\ldots]$ at the unison and octave, and at the duration of a crotchet, and then splitting this again into two discrete canonic entities: in effect, a double canon, each at the octave and unison, but the second canon at the 5th (or 4th below) in relation to the first, and at a duration of 8 bars. The material for the vocal parts either make use of the canonic lines when appropriate and possible, or related extra lines were added to carry the text, to 'float' above the texture. Taking care over pitch content placement meant that a canonic texture would always produce a gently moving pitch cluster at a slow tempo orientated around an overall tonal centre.

(interview)

A primary concern for Aitchison, as seen also for Pickard in relation to the extract in Figure 3 above, was to allow for the 'complex and subtle distortion' brought about by the latency, 'while maintaining a recognizable overall shape - the stepwise, scale-like quality of the melodic contour' (interview). As Aitchison describes,

The music needed to work at varying degrees of 'stretching' of the distance between entries of the canonic lines. I addressed this in two ways: (1) via segmenting the canonic line into sections of clearly delineated and related pitch content; and (2) making sure to separate the segments where the pitch content/qualities changed with periods of silence 
long enough to allow each node to play out their harmonic qualities before the new

segments began.

(interview)

\section{Layered textures}

Each of the previous examples demonstrate in various ways the layering of musical materials to build up larger textures. In the case of Re-Tracing, each layer is a different manifestation of the same underlying melodic line. In the fourth movement of Reuben's Spiritus Telecommunitas, this technique is taken a step further through the layering of multiple short - and independent - musical cells. As shown in Figure 8, slowly evolving string chords are segmented into individual musical lines and distributed throughout the section. These then support a series of motifs in the flutes, brass and voices.

Musical content is derived from a range of sources. Taking inspiration from Gabrielli's antiphonal choral music (see Rofe et al. 2017) and from Charles Ives' layered treatment of instrumental forces, Reuben

[...] wanted to achieve a very gradual move from a more Ivesian type of language, where each instrument is dislocated but ultimately integrated, to a more homophonic sound at the end. I designed a statistical model for the piece whereby the music would gradually morph towards Gabrielli; what you hear right at the end are some Gabrielli chords.

(interview) 
Figure 8: Spiritus Telecommunitas (IV) - Layered cells; b62ff.
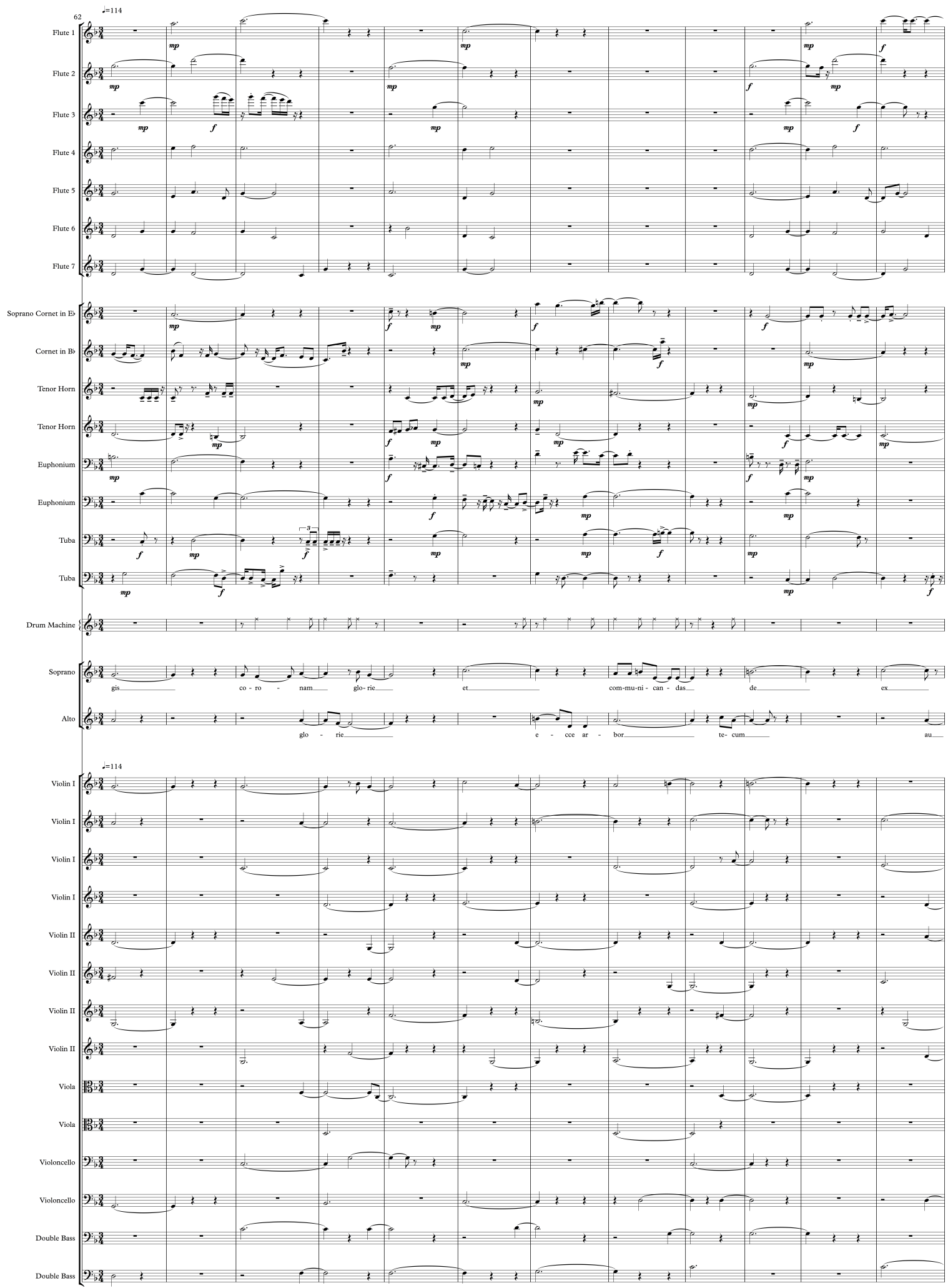
As Reuben goes on to explain,

I wanted to convey the type of information that lives in the internet and how it gets dislocated. So what I wanted was each instrumental force to have its own character. What I did with the brass section, for example, is that I made an algorithm that looks for brass band music online and then downloads it into a database: I had a huge database of brass band material. I then developed a matching algorithm whereby the computer would look into the brass band database trying to match phrases to the original Gabrielli; it would take these little phrases and insert them into the original.

(interview)

The resultant Ivesian texture is one in which these short phrases are layered on top of each other, creating a sound mass formed from multiple individual components. As with earlier examples, this means that, when realized in a latency-rich environment, the precise onset time of each individual cell is less important than the global effect: a layered soundmass that gradually morphs into Gabrielli.

\section{Improvised rhythms}

One final approach to the challenge of latency, as explored by both Pickard and Reuben at various points in their works, is the use of free-time notation. Figure 9 shows an extract from Pickard's In Sea-Cold Lyonesse, in which strings are asked to 'repeat independently in free rhythm, varying the pattern constantly'. This again avoids the need for precise alignment of gestures, creating a freely mobile soundmass over which the voices are set. For Pickard, this more contemporary notation not only sought to address the challenge of latency: 
Figure 9: In Sea-Cold Lyonesse - Free-time rhythmic notation; b87ff.

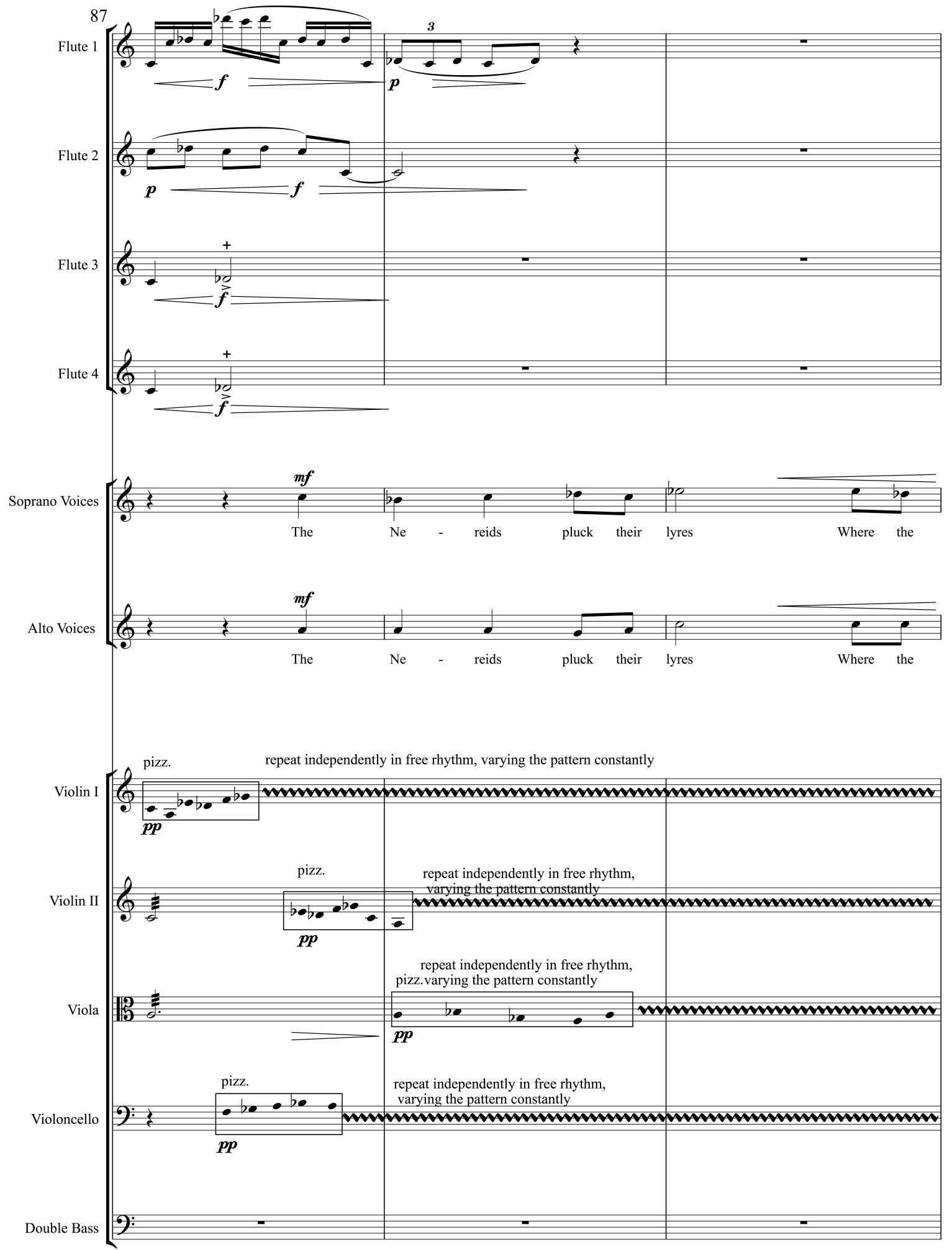


Also there was an educative function here. I knew that we were going to have some young inexperienced players, and I wanted to introduce them to a way of notation that they might not have come across before, and to do this in quite a friendly way. It's more enjoyable to play like this than to play something that is full of quintuples and sextuples and then you worry about whether you are going to get it right and then nobody hears it. It's the eternal sort of battle that you have in modernism between over-notation and under-notation.

(interview)

\section{Conclusions - composing for the medium}

As discussed in Prior (2017) in this special issue, Online Orchestra - and indeed telematic performance more generally - can be considered a medium, complete with its own idiosyncrasies and affordances (see also Braasch 2009). In the case of Online Orchestra, this medium comes with a very specific, and idiosyncratic, rule system that stems from its latency-control programme. But, as Reuben notes,

It is like any other medium in the sense you have to reflect on how to make something successful or work for that medium. As a composer, I tend to look at the medium as being really important. So, for example, I don't just write a string quartet, I would write for a string quartet. In a way I think composers are used to this limitation and will be able to adapt to a medium.

(interview)

And as exemplified by the three works commissioned for Online Orchestra, great musical variety is possible in this medium: the three pieces demonstrate distinct differences not just in their narrative and content, but also in their technical difficulty to 
perform, and their different balances of traditionalism and modernism with respect to harmony and form. Within that variety can also be seen recurrent solutions to the challenge of latency: distributed textures; polyrhythms, ostinati and canons; blurred transitions; slow rates of harmonic change; layered textures; and semi-improvised rhythmic notation.

Aside from the specific issue of latency, a more general challenge of a telematic performance environment is the distributed nature of its musicians. Whilst this distance might be mitigated in part through the use of more immersive technologies, or highquality audio and video streams, the experience will never feel the same as traditional, 'offline' performance, where musicians interact in person. This sense of distance was explored directly by composers, as Pickard explains:

There was something about distance that I wanted to explore in a poetic sense, because there is this wonderful paradoxical sense that the people are not physically present, but they are absolutely present and interacting in a similar way that they would if they were in the same room. And that's a remarkable and a unique thing, something that couldn't have happened in any other stage in history. And yet you cannot avoid the fact that they are not there: it's this 'tyranny of distance'. It has that sense of loss, and of poignancy and of distance. And I had defined some sort of way of projecting that in poetic terms.

(interview)

For Pickard that poetic exploration comes in the form of the underlying narrative behind In Sea-Cold Lyonesse and also in specific musical gestures such as that of b160ff (see Figure 3, above), where musicians call to one another between nodes. For Aitchison, this technique is taken a step further. As shown in Figure 10, as the canonic texture grows materials begin not only to layer across nodes, but also are passed around between nodes: waves of activity sweep through the different locations; a single musical 
Figure 10: Re-Tracing - Passing of material between nodes; b25ff.
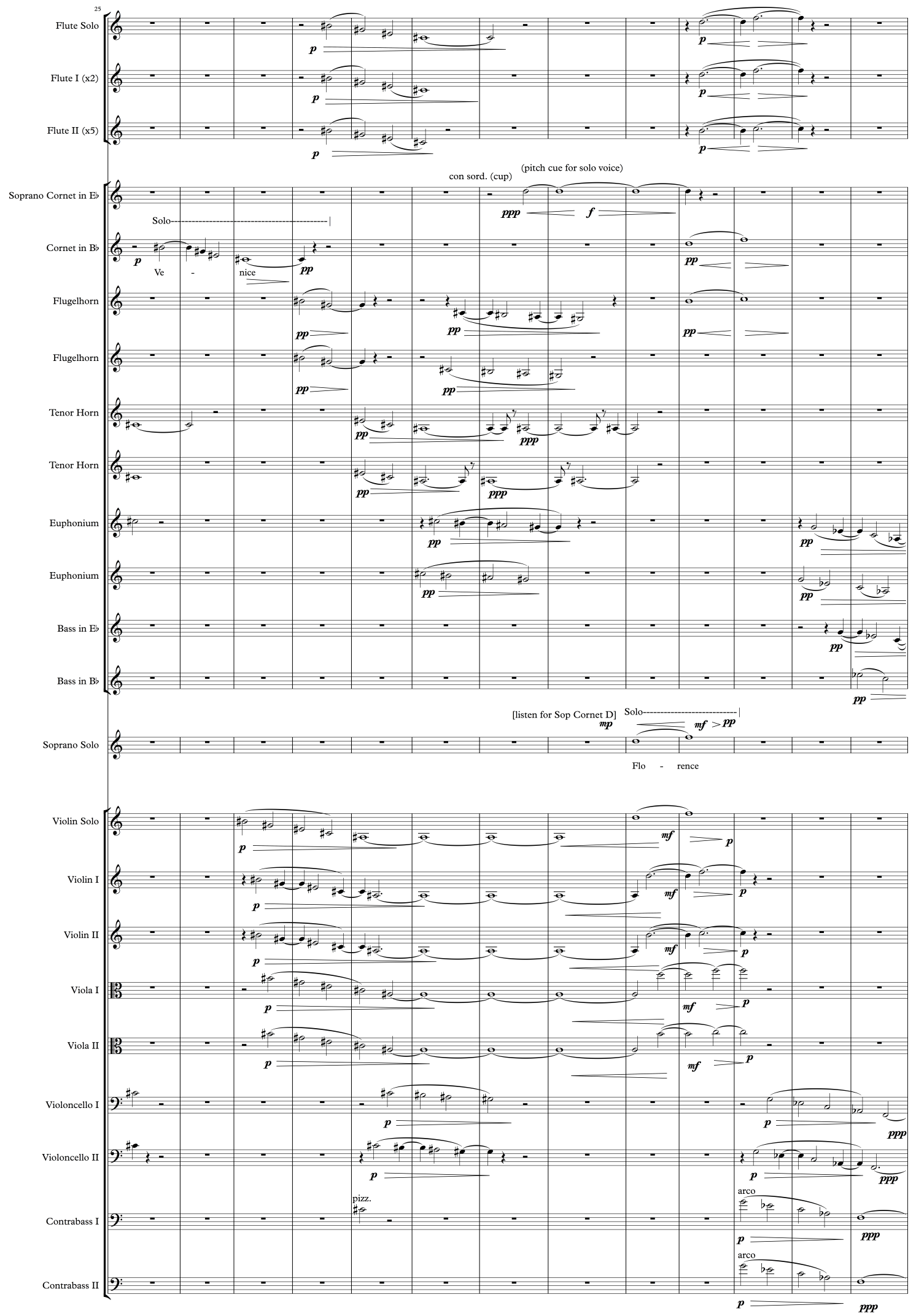
idea travelling around Cornwall. As such, the real potential of telematic performance becomes apparent: this music does not try to overcome the 'problem' of distance or latency; it creatively engages with distance, enabling musical outcomes that would not otherwise be possible in the real, offline world.

This transition in thinking, from the limitations of the telematic medium to its new possibilities, is crucial. As Braasch has written, 'new and better design goals for telematic music collaborations [can be enabled] by treating telematic systems as a new class of musical instruments' (2009: 421). This in turn extends to the compositional domain: the new types of musical possibilities enabled through the telematic medium are still in their infancy, and there is great scope for composers to find their voice - and develop new types of music - in this environment. What the three compositions commissioned for Online Orchestra demonstrate is that this search for a musical language does not need to depart entirely from traditional approaches to composition: music that is orchestral, notated, conducted and that attempts an educative function is indeed possible in a latency-rich, telematic environment. Latency, far from being a hurdle to overcome, becomes an opportunity to embrace.

\section{References}

Aitchison, J. (2016), written report on Online Orchestra, 20 January.

Barbosa, A. (2003), 'Displaced soundscapes: A survey of network systems for music and sonic art creation', Leonardo Music Journal, 13:1, pp. 53-59. 
Braasch, J. (2009), 'The telematic music system: Affordances for a new instrument to shape the music of tomorrow', Contemporary Music Review, 28:4, pp. 421-32.

Chafe, C. (2009), 'Tapping into the Internet as an acoustical/musical medium', Contemporary Music Review, 28:4\&5, pp. 413-20.

Deal, S. and Burtner, M. (2011), 'Auksalaq, a telematic opera', Proceedings of the International Computer Music Conference 2011, Huddersfield, UK, 31 July-5 August, Michigan: University of Michigan.

Driessen, P., Darcie, T. and Pillay, B. (2011), 'The effects of network delay on tempo in musical performance', Computer Music Journal, 35:1, pp. 76-89.

Fields, K. (2012), ‘Syneme: Live’, Organised Sound, 17:1, pp.1-10.

Freeman, J., Ramakrishnan, S., Varnik, K. (2005), 'The architecture of auracle: A voicecontrolled, networked sound instrument', Proceedings of the International Computer Music Conference 2005, Barcelona, Spain, 4-10 September, Michigan: University of Michigan.

Geelhoed, E., Prior, D. and Rofe, M. (2017), 'Designing a system for Online Orchestra: Microphone evaluation and cost-benefit analysis', Journal of Music, Technology and Education, 10: 2-3, pp. 213-30.

Hamilton, R., Caceres, J., Nanou, C. and Platz, C. (2011), 'Multi-modal musical environments for mixed-reality performance', Multimodal User Interfaces, 4:3-4, pp. $147-56$.

Handberg, L. and Jonsson, A. (2005), 'Community building through cultural exchange in mediated performance events', in P. Hernwall (ed.), The Virtual-A Room Without Borders?, Södertörn: Södertörn College University. 
Kim-Boyle, D. (2008), 'Network music's play, engagement and the democratization of performance', Proceedings of New Interfaces for Musical Expression Conference, Geneva, Italy, 5-7 June, Copenhagen: Aalborg University.

Mills, R. (2010), 'Dislocated sound: A survey of improvisation in networked audio platforms', Proceedings of the 2010 Conference on New Interfaces for Musical Expression, Sydney, Australia, 15-18 June, Copenhagen: Aalborg University.

Mizuno, M. (2011), 'On the music through network: Telematic performance of “telequantum", Proceedings of Asia Computer Music Project 2011, Tokyo, Japan, 16-18 December, Nagoya: Nagoya City University.

Pickard, J. (2016), face-to-face interview with M. Rofe, 5 March, Bristol.

Prior, D. (2017), 'The network as niche', Journal of Music, Technology and Education, 10: 2-3, pp. 289-303.

Prior, D., Reeder, P., Rofe, M., Biscoe, I. and Murray, S. (2017), 'Designing a system for Online Orchestra: Peripheral equipment', Journal of Music, Technology and Education, 10: 2-3, pp. 197-212.

Reuben, F. (2016), Skype interview with M. Rofe, 1 March., Falmouth \& York. Rofe, M. and Reuben, F. (2017), 'Telematic performance and the challenge of latency', Journal of Music, Technology and Education, 10: 2-3, pp. 167-84.

Rofe, M., Murray, S. and Parker, W. (2017), 'Online Orchestra: Connecting remote communities through music', Journal of Music, Technology and Education, 10: 23, pp. 147-66.

Rothko, M. (1934), 'Our July art forum', New York Times, July part 7, p. X7. 
Weaver, S. (2011), 'Latency: Music composition and technology solutions for perception of synchrony in "ResoNations 2010: An International Telematic Music Concert for Peace", Masters thesis, New York: New York University.

Whalley, I. (2015), 'Developing telematic electroacoustic music: Complex networks, machine intelligence and affective data stream sonification', Organised Sound, 20:1, pp. 90-98.

Notes

1. Map exported from Google Maps.

Michael Rofe and Erik Geelhoed have asserted their rights under the Copyright, Designs and Patents Act, 1988, to be identified as the authors of this work in the format that was submitted to Intellect Ltd. 